\title{
The bowel, the genitourinary tract, and infective endocarditis
}

\author{
RICHARD BAYLISS, CYRIL CLARKE, C M OAKLEY, W SOMERVILLE, \\ A G W WHITFIELD, S E J YOUNG
}

From the Royal College of Physicians Research Unit, the British Cardiac Society, and the Communicable Diseases Surveillance Centre, London

SUMMARY Of 582 episodes of infective endocarditis 75 were attributable to organisms normally resident in the bowel and 12 others were associated with alimentary tract operations, investigations, or disease. The mean age of the 87 patients in this particular group was higher (59.7. years) than that of all the patients with infective endocarditis ( 51.4 years). As far as could be ascertained $41 \%$ had no pre-existing cardiac abnormality, and in a little under a half no predisposing event to initiate the illness was apparent. Where the portal of entry of the organism to the blood stream was evident it was slightly more often in the genitourinary than the alimentary tract.

Bowel organisms are no less important than those associated with the teeth in causing infective endocarditis. It is suggested that in all those patients with known cardiac abnormalities and possibly in those over the age of 60 with normal hearts antibiotic cover should be considered when they undergo genitourinary or alimentary tract surgery or instrumentation.

The prognosis of infective endocarditis has improved dramatically since the advent of antibiotic treatment $^{1-13}$ and more recently as a result of emergency valve replacement. ${ }^{35891213}$ In the best centres the mortality rate is now apparently less ${ }^{814}$ than the widely quoted figure of $30 \% .451013-15$ Nevertheless, the prevalence of the disease is probably increasing and it remains a serious threat to life in all who suffer from it. Doubtless there will be further reductions in mortality with advances in cardiology, microbiology, and surgery, but the major effort must be in prevention. Those with rheumatic, congenital, or degenerative heart disease, with prosthetic valves ${ }^{6} 79^{1216^{-1}}$ or who have undergone other cardiac surgery, ${ }^{47}$ and those with a history of infective endocarditis ${ }^{41216}$ are at especial risk and should have antibiotic prophylaxis if they need dental treatment or investigatory or therapeutic procedures related to the alimentary, genitourinary, or respiratory tracts. In addition, the immunosuppressed, those addicted to alcohol, and those with diabetes ${ }^{3}$ or malignant disease ${ }^{4}$ are particularly vulnerable. Nevertheless, one tenth of patients with infective endocarditis have a pre-existing cardiac

Requests for reprints to Royal College of Physicians Research Unit, 11 St Andrews Place, London NW1 4LE.

Accepted for publication 1 November 1983 abnormality which is not known to either them or their doctor, ${ }^{20}$ and it is also becoming increasingly clear that a large proportion of patients with infective endocarditis have clinically normal hearts before the onset of their illness, ${ }^{6-913162021}$ which are important factors that influence the application of any planned prophylactic regimen.

In a few patients with infective endocarditis the portal of entry of the causal organism is beyond dispute. In the vast majority, however, the mechanism or route by which the organism gains entry to the blood stream is unknown or unproved. Oral organisms are the most common, and dental surgery has been suspected for many years; although this is doubtless the cause in some patients, definite proof is lacking, and the proportion so attributable is probably small. ${ }^{22}$ Other infections are thought to arise from the alimentary, genitourinary, or respiratory tracts or from the skin but again the evidence, though strong, is largely circumstantial. In fact the only group in which causation appears certain is in the right sided endocarditis of drug addicts.

A recent investigation conducted jointly by the Royal College of Physicians Research Unit and the British Cardiac Society and related especially to the dental prophylaxis of infective endocarditis has provided the largest series of patients yet studied. ${ }^{2022}$ 
This investigation concerned those patients in whom the causal organism's normal, but not exclusive, habitat was the alimentary tract and those in whom the illness followed some alimentary tract investigation, operation, or disease. Could infective endocarditis have been prevented in this not inconsiderable group?

\section{Methods}

Members of the British Cardiac Society and fellows and members of the Royal College of Physicians in the United Kingdom were asked to complete a proforma in respect of all patients with infective endocarditis under their care during 1981 and 1982. The information requested included inter alia the causal organism, whether there was any known or unknown preexisting cardiac abnormality, whether there had been any dental, other surgical, or investigative procedures or injury during the three months before the onset of illness, and if so whether antibiotic cover had been provided, and details of any other relevant factors.

\section{Results}

Proformas in respect of 582 episodes of infective endocarditis occurring in 577 patients were received, five patients having had a second episode within the two year period. The age range of the patients was from 2 to 87 (mean age 51.4) years and there was a male preponderance (ratio $2: 1$ ).

Among the 582 episodes the evidence suggested that the alimentary or genitourinary tracts may have been responsible in $87(15 \%)$. The causal organism was an unspeciated enterococcus in 17, Streptococcus faecalis in 14, Streptococcus bovis in 27 (Table 1), Escherichia coli and other Gram negative bacilli in 11, and Streptococcus durans or group D streptococci in six (Table 2). In addition there were 12 patients in whom the illness was temporally related to some alimentary tract disease or operation but in whom the dominant habitat of the causal organism was not the bowel (Table 3). Similarly details are given (but not otherwise considered here) of patients whose infective endocarditis was not caused by bowel organisms but whose illness was associated with genitourinary disease, investigation, or surgery (Table 3 ).

The twofold male preponderance in the group as a whole was the same in the 87 patients whose infective endocarditis may have arisen from the alimentary tract, but the mean age was higher in the latter (59.7 compared with 51.4 years). Ten of the 87 patients died, a slightly smaller proportion than that in the total 577 patients.

Of the 87 patients, $36(41 \%)$ had, as far as could be ascertained, no pre-existing cardiac abnormality, a proportion exactly the same as in the total 577 patients. The valves affected did not differ in the alimentary tract group from all those examined, the aortic valve being affected in 29 patients and the mitral valve in 23 .

\section{Discussion}

Table 1 shows that of the 17 cases due to unspeciated enterococci one was associated with gall bladder disease, one followed an appendicectomy and one a gastrointestinal infection, one had undergone a total pancreatectomy and was a diabetic and dependent on alcohol, and one had had a dental filling without antibiotic cover within the previous three months when his heart was thought to be normal. Three cases arose from the genitourinary tract: one after a transurethral prostatic resection, one after urethral dilatation, and one in association with a urinary tract infection. In another patient a lacerated hand was the portal of entry, but in the remaining eight patients there was no indication of how the organism gained entrance to the blood stream. These findings in respect of enterococcal infections are similar to those of other workers. ${ }^{21} 23$ The fact that enterococci commonly colonise the anterior urethra, the vagina, and the cervix explains the cases arising from the genitourinary tract.

In the $14 S$ faecalis infections (Table 1) the causal factor was evident in all but two patients, one of whom had had a previous attack of infective endocarditis. Three infections followed an alimentary tract operation (hemicolectomy, haemorrhoidectomy, and surgery for a gangrenous femoral hernia). One case followed dental sepsis and one a Colles fracture and may have arisen from it or from the anaesthetic for its reduction. Five cases arose from the genitourinary tract (prostatectomy for carcinoma, cystoscopy, vaginal hysterectomy, insertion of a ring pessary, urinary tract infection), and one case developed during the thirty third week of pregnancy. Infective endocarditis during pregnancy has been reported, 1524 and $S$ faecalis, like unspeciated enterococci, often colonise the lower genitourinary tract and perineum. In 15 of the 27 cases due to $S$ bovis (Table 1), however, there was no indication of why the infection developed except that one of the 15 patients had had a previous episode of infective endocarditis and one had a prosthetic mitral valve. Six cases were associated with alimentary tract disease or procedures (gastric polyp, tubocellular adenomata of colon, closure of colostomy, liver biopsy in a patient who probably had a carcinoma, and diverticular disease in two patients). In a further three patients dental sepsis was present, and one had had scaling within two months of the onset of illness with antibiotic cover. Of the remaining two patients, one was receiving prednisone for asthma 
Table 1 Details of patients with infective endocarditis due to various bowel organisms

\begin{tabular}{|c|c|c|c|c|c|c|}
\hline $\begin{array}{l}\text { Case } \\
\text { No }\end{array}$ & Sex & $\begin{array}{l}\text { Age } \\
(y r)\end{array}$ & Previous cardiac abnormality & $\begin{array}{l}\text { Valve } \\
\text { affected }\end{array}$ & $\begin{array}{l}\text { Portal of entry or other } \\
\text { causal factor }\end{array}$ & Outcome \\
\hline \multicolumn{7}{|c|}{ Unspeciated enterococci } \\
\hline $\begin{array}{r}6 \\
24\end{array}$ & $\begin{array}{l}\mathbf{M} \\
\mathbf{M}\end{array}$ & $\begin{array}{l}75 \\
61\end{array}$ & $\begin{array}{l}\text { None } \\
\text { Congenital aortic stenosis and } \\
\text { previous AVR }\end{array}$ & $\begin{array}{l}\text { Aortic } \\
\text { Aortic }\end{array}$ & $\begin{array}{l}\text { TUR } \\
\text { None }\end{array}$ & $\begin{array}{l}\text { Died } \\
\text { Died }\end{array}$ \\
\hline 36 & $\mathbf{M}$ & 61 & Rheumatic heart disease & Mitral & $\begin{array}{l}\text { Gastrointestinal infection } 3 \text { weeks } \\
\text { before onset }\end{array}$ & \\
\hline 37 & $\mathbf{M}$ & 38 & None & NS & $\begin{array}{l}\text { Diabetic. Alcoholic. Had had total } \\
\text { pancreatectomy }\end{array}$ & \\
\hline $\begin{array}{l}39 \\
59\end{array}$ & $\begin{array}{l}M \\
M\end{array}$ & $\begin{array}{l}61 \\
18\end{array}$ & $\begin{array}{l}\text { Rheumatic heart disease } \\
\text { None }\end{array}$ & $\begin{array}{l}\text { NS } \\
\text { Aortic }\end{array}$ & $\begin{array}{l}\text { None } \\
\text { Dental filling within } 3 \text { months without } \\
\text { antibiotic cover }\end{array}$ & \\
\hline $\begin{array}{l}85 \\
103 \\
106 \\
126\end{array}$ & $\begin{array}{l}\mathbf{M} \\
\mathbf{M} \\
\mathbf{M} \\
\mathbf{F}\end{array}$ & $\begin{array}{l}62 \\
60 \\
44 \\
71\end{array}$ & $\begin{array}{l}\text { Previous AVR for aortic stenosis } \\
\text { Previous AVR for aortic stenosis } \\
\text { Bicuspid aortic valve } \\
\text { Known cardiac abnormality nature } \\
\text { uncertain }\end{array}$ & $\begin{array}{l}\text { Aortic } \\
\text { Aortic } \\
\text { Aortic } \\
\text { NS }\end{array}$ & $\begin{array}{l}\text { None } \\
\text { Lacerated thumb } \\
\text { None } \\
\text { Urinary tract infection }\end{array}$ & Died \\
\hline $\begin{array}{l}127 \\
243 \\
245 \\
331 \\
345 \\
413 \\
440\end{array}$ & $\begin{array}{l}\mathrm{F} \\
\mathrm{M} \\
\mathrm{F} \\
\mathrm{M} \\
\mathrm{M} \\
\mathrm{F} \\
\mathrm{F}\end{array}$ & $\begin{array}{l}46 \\
76 \\
29 \\
71 \\
63 \\
58 \\
80\end{array}$ & $\begin{array}{l}\text { None } \\
\text { Rheumatic heart disease } \\
\text { Rheumatic heart disease } \\
\text { None } \\
\text { None } \\
\text { None } \\
\text { None } \\
\end{array}$ & $\begin{array}{l}\text { Aortic } \\
\text { Mitral } \\
\text { Aortic } \\
\text { NS } \\
\text { Aortic } \\
\text { NS } \\
\text { NS } \\
\text { faecalis }\end{array}$ & $\begin{array}{l}\text { None } \\
\text { Urethral dilatation } \\
\text { Appendectomy } \\
\text { None } \\
\text { Gall bladder disease } \\
\text { None } \\
\text { None }\end{array}$ & \\
\hline 13 & $\mathbf{M}$ & 60 & \multicolumn{2}{|c|}{$\begin{array}{r}\text { Streptococcus faecalis } \\
\text { Aortic }\end{array}$} & $\begin{array}{l}\text { Right hemicolectomy for } \\
\text { angiodysplasia }\end{array}$ & Died \\
\hline $\begin{array}{r}18 \\
87 \\
146 \\
157 \\
204\end{array}$ & $\begin{array}{l}\mathbf{F} \\
\mathbf{F} \\
\mathbf{F} \\
\mathbf{M}\end{array}$ & $\begin{array}{l}58 \\
27 \\
73 \\
47 \\
68\end{array}$ & $\begin{array}{l}\text { Previous IE } \\
\text { VSD } \\
\text { None } \\
\text { Rheumatic heart disease } \\
\text { None }\end{array}$ & $\begin{array}{l}\text { Mitral } \\
\text { VSS } \\
\text { Mitral } \\
\text { NS } \\
\text { Mitral and } \\
\quad \text { aortic }\end{array}$ & $\begin{array}{l}\text { Previous IE } \\
33 \text { weeks' pregnant } \\
8 \text { weeks after Colles fracture } \\
\text { After operation for haemorrhoids } \\
\text { None }\end{array}$ & $\begin{array}{l}\text { Died } \\
\text { Died }\end{array}$ \\
\hline 217 & $\mathbf{F}$ & 73 & Aortic stenosis & Aortic & $\begin{array}{l}\text { After operation for gangrenous } \\
\text { femoral hernia }\end{array}$ & \\
\hline $\begin{array}{l}221 \\
309 \\
320 \\
435 \\
437 \\
481\end{array}$ & $\begin{array}{l}\mathbf{M} \\
\mathbf{M} \\
\mathbf{F} \\
\mathbf{M} \\
\mathbf{M}\end{array}$ & $\begin{array}{l}71 \\
\text { NS } \\
77 \\
79 \\
66 \\
71\end{array}$ & $\begin{array}{l}\text { Known murmur } \\
\text { None } \\
\text { Known mitral incompetence } \\
\text { None } \\
\text { Had had MVR } \\
\text { Mitral valve prolapse }\end{array}$ & $\begin{array}{l}\text { Aortic } \\
\text { NS } \\
\text { Mitral } \\
\text { NS } \\
\text { Mitral } \\
\text { Mitral }\end{array}$ & $\begin{array}{l}\text { None } \\
\text { After vaginal hysterectomy } \\
\text { Urinary tract infection } \\
\text { After insertion of a ring pessary } \\
\text { After cystoscopy } \\
\text { After prostatectomy for carcinoma of } \\
\text { prostate }\end{array}$ & \\
\hline 506 & $\mathbf{M}$ & 59 & $\begin{array}{l}\text { Degenerative heart disease } \\
\text { Streptococcu }\end{array}$ & $\begin{array}{l}\text { Aortic } \\
\text { s bovis }\end{array}$ & Dental sepsis & \\
\hline 16 & $\mathbf{F}$ & 61 & Congenital heart disease & $\begin{array}{l}\text { Congenital } \\
\text { defect }\end{array}$ & Dental sepsis & \\
\hline $\begin{array}{l}21 \\
57\end{array}$ & $\underset{M}{F}$ & $\begin{array}{l}\text { NS } \\
34\end{array}$ & $\begin{array}{l}\text { Rheumatic heart disease } \\
\text { Aortic incompetence }\end{array}$ & $\begin{array}{l}\text { NS } \\
\text { Aortic and } \\
\text { mitral }\end{array}$ & $\begin{array}{l}\text { None } \\
\text { None }\end{array}$ & \\
\hline $\begin{array}{l}105 \\
112 \\
116 \\
150 \\
171 \\
175 \\
182 \\
233\end{array}$ & $\begin{array}{l}\mathbf{F} \\
\mathbf{M} \\
\mathbf{M} \\
\mathbf{M} \\
\mathbf{M} \\
\mathbf{M} \\
\mathbf{F}\end{array}$ & $\begin{array}{l}54 \\
42 \\
43 \\
44 \\
62 \\
77 \\
38 \\
46\end{array}$ & $\begin{array}{l}\text { None } \\
\text { Rheumatic heart disease } \\
\text { None } \\
\text { None } \\
\text { Rheumatic heart disease } \\
\text { Mitral incompetence } \\
\text { Bicuspid aortic valve } \\
\text { Rheumatic heart disease, } \\
\text { previous MVR }\end{array}$ & $\begin{array}{l}\text { NS } \\
\text { NS } \\
\text { Mitral } \\
\text { NS } \\
\text { NS } \\
\text { Mitral } \\
\text { Aortic } \\
\text { Mitral }\end{array}$ & $\begin{array}{l}\text { Dental sepsis } \\
\text { None } \\
\text { Alcoholism } \\
\text { Dental sepsis } \\
\text { None } \\
\text { Gastric polyp, dental caries } \\
\text { None } \\
\text { None }\end{array}$ & \\
\hline $\begin{array}{l}240 \\
259 \\
273\end{array}$ & $\begin{array}{l}M \\
M \\
M\end{array}$ & $\begin{array}{l}55 \\
68 \\
63\end{array}$ & $\begin{array}{l}\text { None } \\
\text { Mitral incompetence } \\
\text { None }\end{array}$ & $\begin{array}{l}\text { NS } \\
\text { Mitral } \\
\text { Aortic }\end{array}$ & $\begin{array}{l}\text { None } \\
\text { None } \\
\text { Scaling } 5-8 \text { weeks previously } \\
\text { without antibiotic cover }\end{array}$ & Died \\
\hline $\begin{array}{l}327 \\
337 \\
389 \\
418 \\
447 \\
452 \\
461 \\
474 \\
480 \\
490 \\
493 \\
538 \\
545\end{array}$ & $\begin{array}{l}\mathbf{M} \\
\mathbf{M} \\
\mathbf{F} \\
\mathbf{F} \\
\mathbf{M} \\
\mathbf{F} \\
\mathbf{M} \\
\mathbf{F} \\
\mathbf{M} \\
\mathbf{M} \\
\mathbf{F} \\
\mathbf{M} \\
\mathbf{F}\end{array}$ & $\begin{array}{l}61 \\
71 \\
76 \\
61 \\
72 \\
69 \\
61 \\
61 \\
60 \\
48 \\
63 \\
77 \\
80\end{array}$ & $\begin{array}{l}\text { None } \\
\text { Myocardial infarction (1973) } \\
\text { Aortic sclerosis } \\
\text { Mitral incompetence } \\
\text { None } \\
\text { None } \\
\text { None } \\
\text { None } \\
\text { None } \\
\text { None } \\
\text { None } \\
\text { Rheumatic heart disease, IE (1981) } \\
\text { Rheumatic heart disease }\end{array}$ & $\begin{array}{l}\text { Aortic } \\
\text { NS } \\
\text { Aortic } \\
\text { Mitral } \\
\text { Mitral } \\
\text { NS } \\
\text { NS } \\
\text { NS } \\
\text { NS } \\
\text { NS } \\
\text { Aortic } \\
\text { NS } \\
\text { Mitral } \\
\end{array}$ & $\begin{array}{l}\text { None } \\
\text { Closure of colostomy } \\
\text { None } \\
\text { None } \\
\text { None } \\
\text { Diverticulitis } \\
\text { Liver biopsy, probably carcinoma } \\
\text { Prednisone for asthma } \\
\text { Diverticular disease } \\
\text { Two tubocellular adenomata of colon } \\
\text { None } \\
\text { None } \\
\text { None }\end{array}$ & Died \\
\hline
\end{tabular}

TUR, transurethral resection; AVR, aortic valve replacement; VSD, ventricular septal defect; NS, not stated; IE, infective endocarditis; MVR, mitral valve replacement. 
Table 2 Details of patients with infective endocarditis due to $E$ coli, gram negative bacilli, $S$ durans, and group $D$ streptococci

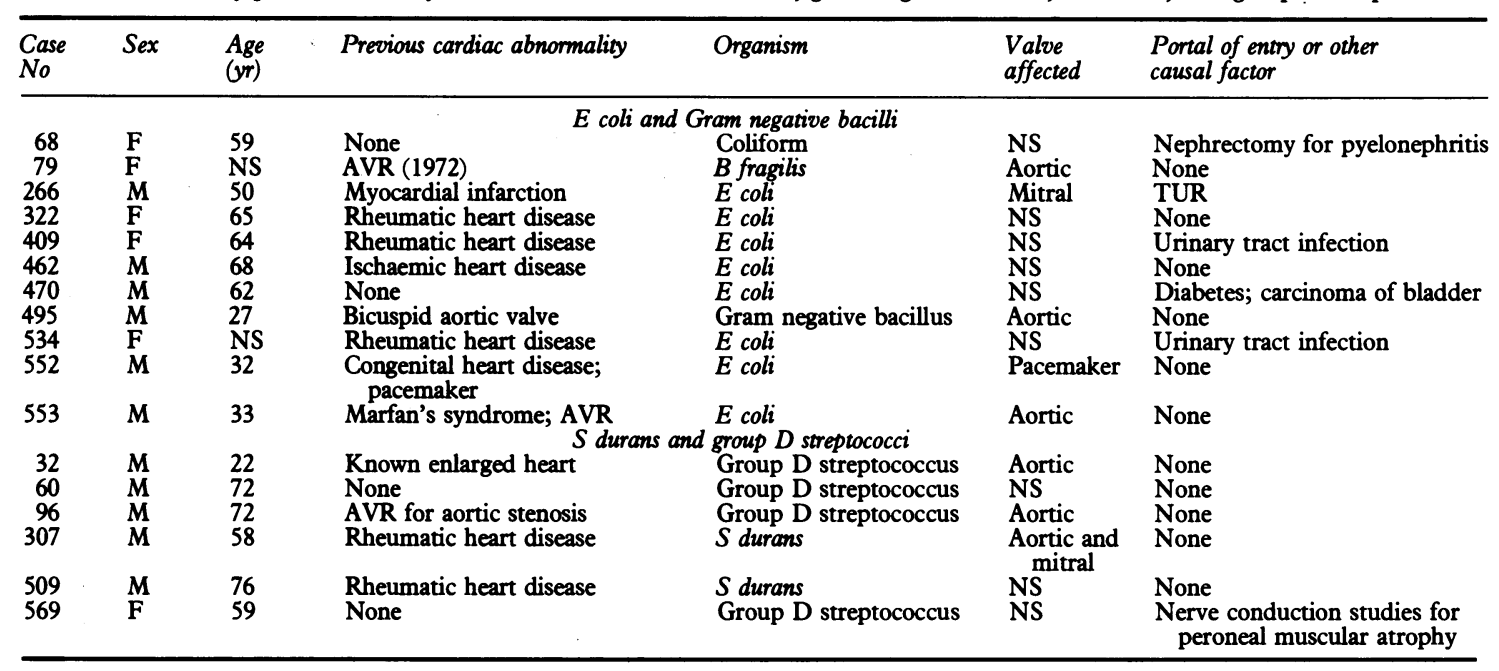

NS, not stated; AVR, aortic valve replacement; TUR, transurethral resection.

Table 3 Details of patients with infective endocarditis apparently related to the alimentary tract or the genitourinary tract

\begin{tabular}{|c|c|c|c|c|c|c|}
\hline $\begin{array}{l}\text { Case } \\
\text { No }\end{array}$ & Sex & $\begin{array}{l}\text { Age } \\
(y r)\end{array}$ & Previous cardiac abnormality & Organism* & $\begin{array}{l}\text { Valve } \\
\text { affected }\end{array}$ & $\begin{array}{l}\text { Portal of entry or other } \\
\text { causal factor }\end{array}$ \\
\hline 114 & . $F$ & 73 & None & $\begin{array}{l}\text { Alimentary tract } \\
\alpha \text { haemolytic streptococcus }\end{array}$ & Aortic & $\begin{array}{l}\text { Cryptogenic cirrhosis; monilial } \\
\text { angular stomatitis }\end{array}$ \\
\hline $\begin{array}{l}119 \\
192 \\
195\end{array}$ & $\begin{array}{l}\mathbf{M} \\
\mathbf{M} \\
\mathbf{M}\end{array}$ & $\begin{array}{l}79 \\
66 \\
65\end{array}$ & $\begin{array}{l}\text { Ischaemic heart disease } \\
\text { Rheumatic heart disease } \\
\text { None }\end{array}$ & $\begin{array}{l}\alpha \text { haemolytic streptococcus } \\
\alpha \text { haemolytic streptococcus } \\
\text { Coagulase negative } \\
\text { staphylococcus }\end{array}$ & $\begin{array}{l}\text { NS } \\
\text { Mitral } \\
\text { NS }\end{array}$ & $\begin{array}{l}\text { Bilateral inguinal herniotomy } \\
\text { Dilatation of oesophageal stricture } \\
\text { Right inguinal herniotomy }\end{array}$ \\
\hline 380 & $\mathbf{M}$ & 27 & Congenital heart disease & Group B streptococcus & $\begin{array}{c}\text { Congenital } \\
\text { lesion }\end{array}$ & $\begin{array}{l}\text { Down's syndrome; } \\
\text { bleeding haemorrhoids }\end{array}$ \\
\hline $\begin{array}{l}381 \\
434\end{array}$ & $\begin{array}{l}\mathrm{F} \\
\mathrm{F}\end{array}$ & $\begin{array}{l}57 \\
63\end{array}$ & $\begin{array}{l}\text { Rheumatic heart disease } \\
\text { Previous MVR and TVR }\end{array}$ & $\begin{array}{l}\alpha \text { haemolytic streptococcus } \\
\text { Negative }\end{array}$ & $\begin{array}{l}\text { Aortic } \\
\text { Tricuspid } \\
\text { and mitral }\end{array}$ & $\begin{array}{l}\text { Gall bladder disease } \\
\text { Gastroscopy; dental sepsis }\end{array}$ \\
\hline $\begin{array}{l}457 \\
473\end{array}$ & $\begin{array}{l}M \\
M\end{array}$ & $\begin{array}{l}71 \\
86\end{array}$ & $\begin{array}{l}\text { None } \\
\text { None }\end{array}$ & $\begin{array}{l}\alpha \text { haemolytic streptococcus } \\
S \text { mutans }\end{array}$ & $\begin{array}{l}\text { Mitral } \\
\text { Aortic }\end{array}$ & $\begin{array}{l}\text { Gastroscopy } \\
\text { Cholecystectomy for empyema of } \\
\text { gall bladder }\end{array}$ \\
\hline $\begin{array}{l}485 \\
512 \\
578\end{array}$ & $\begin{array}{l}\mathbf{M} \\
\mathbf{M} \\
\mathbf{M}\end{array}$ & $\begin{array}{l}60 \\
78 \\
52\end{array}$ & $\begin{array}{l}\text { None } \\
\text { Mitral incompetence } \\
\text { None }\end{array}$ & $\begin{array}{l}S \text { sanguis } \\
S \text { mutans } \\
\text { Group B streptococcus } \\
\text { Genitourinary tract }\end{array}$ & $\begin{array}{l}\text { Mitral } \\
\text { Mitral } \\
\text { NS }\end{array}$ & $\begin{array}{l}\text { Surgery for rectal carcinoma } \\
\text { Carcinoma of rectum } \\
\text { Liver failure }\end{array}$ \\
\hline $\begin{array}{r}1 \\
41\end{array}$ & $\begin{array}{l}\mathbf{M} \\
\mathbf{M}\end{array}$ & $\begin{array}{l}75 \\
74\end{array}$ & $\begin{array}{l}\text { Rheumatic heart disease } \\
\text { None }\end{array}$ & $\begin{array}{l}S \text { viridans } \\
\text { Negative }\end{array}$ & $\begin{array}{l}\text { NS } \\
\text { Aortic }\end{array}$ & $\begin{array}{l}\text { Urinary tract infection } \\
\text { Within one month of retropubic } \\
\text { prostatectomy }\end{array}$ \\
\hline 131 & $M$ & 57 & $\begin{array}{l}\text { Infective endocarditis and valve } \\
\text { replacement (1973) }\end{array}$ & Staphylococcus aureus & NS & $\begin{array}{l}\text { Urethral dilatation; urinary tract } \\
\text { infection }\end{array}$ \\
\hline $\begin{array}{l}144 \\
199 \\
209\end{array}$ & $\begin{array}{l}\mathbf{F} \\
\mathbf{M}\end{array}$ & $\begin{array}{l}37 \\
55 \\
71\end{array}$ & $\begin{array}{l}\text { None } \\
\text { Aortic valve replacement (1973) } \\
\text { None }\end{array}$ & $\begin{array}{l}S \text { viridans } \\
\text { Negative } \\
\text { Proteus sp }\end{array}$ & $\begin{array}{l}\text { Mitral } \\
\text { Aortic } \\
\text { Mitral }\end{array}$ & $\begin{array}{l}\text { Followed parturition } \\
\text { Urinary tract infection } \\
\text { Pyelonephritis; diabetes } \\
\text { mellitus; proteus sp in urine }\end{array}$ \\
\hline $\begin{array}{l}210 \\
292\end{array}$ & $\begin{array}{l}\mathrm{F} \\
\mathrm{F}\end{array}$ & $\begin{array}{l}38 \\
72\end{array}$ & $\begin{array}{l}\text { None } \\
\text { None }\end{array}$ & $\begin{array}{l}\text { S viridans } \\
\text { Staph aureus }\end{array}$ & $\begin{array}{l}\text { Aortic } \\
\text { NS }\end{array}$ & $\begin{array}{l}22 \text { weeks pregnant } \\
\text { Within one month of repair of } \\
\text { vaginal prolapse; diabetes; } \\
\text { myxoedema }\end{array}$ \\
\hline 334 & $\mathbf{M}$ & 36 & None & $S$ sanguis & $\begin{array}{l}\text { Aortic and } \\
\text { mitral }\end{array}$ & $\begin{array}{l}\text { Renal calculus and colic; } \\
\text { ?Marfan's syndrome }\end{array}$ \\
\hline 431 & $\mathbf{M}$ & 65 & $\begin{array}{l}\text { Bicuspid aortic valve } \\
\text { (not known) }\end{array}$ & Pseudomonas aeruginosa & Aortic & Urinary tract infection \\
\hline 433 & $\mathbf{M}$ & 68 & Calcific aortic stenosis & Staphylococcus albus & Aortic & $\begin{array}{l}\text { Followed transurethral prostatic } \\
\text { resection; rheumatoid arthritis }\end{array}$ \\
\hline 522 & F & 63 & $\begin{array}{l}\text { Aortic and mitral valve } \\
\text { replacements for rheumatic } \\
\text { heart disease (1981) }\end{array}$ & $S$ viridans & $\begin{array}{l}\text { Aortic and } \\
\text { mitral }\end{array}$ & $\begin{array}{l}\text { Carcinoma of cervix; } \\
\text { dilatation and curettage and } \\
\text { radiotherapy }\end{array}$ \\
\hline
\end{tabular}

TUR, transurethral resection; MVR, mitral valve replacement; TVR, tricuspid valve replacement; NS, not stated.

${ }^{\star} \alpha$ haemolytic streptococcus was previously known as $S$ viridans. 
and the other was dependent on alcohol.

Table 2 shows the $11 E$ coli and other Gram negative infections. In five cases the organism appeared to come from the urinary tract, while in the remaining six there was no indication of how it gained entrance to the blood stream. $E$ coli and other Gram negative bacilli are of course often responsible for urinary tract infection. Of the six cases due to group D streptococci or $S$ durans (Table 2), one followed nerve conduction studies, but in the other five the portal of entry was not known, although one of the five had a prosthetic valve.

By definition all patients shown in Table 3 had some alimentary tract disorder or had undergone an operation or investigation: liver disease (two), gall bladder disease (two), rectal carcinoma (two), herniotomy (two), haemorrhoids (one), and dilatation of an oesophageal stricture (one). In eight of the 12 cases the organism was of the Streptococcus viridans group, in two a group B streptococcus (considered by some to be a bowel organism), in one a coagulase negative staphylococcus, and in one the culture was negative. Of the 75 cases of infective endocarditis due to organisms normally present in the bowel, there were therefore 12 that appeared to arise from the alimentary tract and 15 from the genitourinary tract, while in $\mathbf{4 0}$ the portal of entry was not known.

In addition to dental procedures many investigations, diseases, and operations of the alimentary tract are known to be associated with transient symptomless bacteraemia.

Bacteraemia has been reported after upper gastrointestinal endoscopy ${ }^{25}$ and after endoscopic retrograde cholangiopancreatography, ${ }^{26}$ but in none of these cases did infective endocarditis develop. In two of the patients (cases 434 and 457), however, infective endocarditis followed gastroscopy. Case 434 had prosthetic mitral and tricuspid valves and dental sepsis; case 457 had no pre-existing cardiac abnormality but the causal organism was an $\alpha$ haemolytic streptococcus (previously termed $S$ viridans). Gastroscopy may be associated with considerable mucosal trauma. Buchman and Bergland reported positive blood cultures after sigmoidoscopy in 13 of 100 patients but thought that most, if not all, were due to contaminants. ${ }^{27}$ Le Frock and his colleagues ${ }^{28}$ and Weinstein, ${ }^{29}$ however, found transient symptomless bacteraemia in $9.5 \%$ of 200 patients after sigmoidoscopy, but this was not confirmed by Engeling and his coworkers. ${ }^{30}$ Moreover, blood cultures after colonoscopy with or without biopsy have been negative ${ }^{31}$ unless associated with colonic carcinoma, ${ }^{32}$ itself well known to be associated with infective endocarditis. ${ }^{33}$ Two of our 90 patients had rectal carcinoma (cases 485 and 512 ).

Transient symptomless bacteraemia has been reported after a barium enema ${ }^{29} 34$ and even after a normal bowel action. Bacteraemia ${ }^{35} 36$ but not infective endocarditis has been reported after percutaneous liver biopsy and after transrectal prostatic biopsy. ${ }^{37}$ One of the patient's symptoms of infective endocarditis began after liver biopsy (case 461), but he probably had carcinomatosis.

There have been many reports linking $S$ bovis endocarditis with carcinoma of the colon ${ }^{38-40}$ and other colonic disorders, ,1-43 $^{-4 n}$ in some the endocarditis has presented before any symptoms of the colonic lesion have been evident. ${ }^{39}$ Although 27 of the 75 patients with endocarditis caused by bowel organisms had $S$ bovis infections, only six had any abnormality of the alimentary tract.

Disease of the biliary tract is often associated with bacteraemia, and there have been reports of infective endocarditis arising from it particularly when surgical intervention is necessary. 151644 Gall bladder disease (case 345 ) and cholecystectomy (case 473 ) were the only examples among the 87 patients.

There have been excellent studies of enterococcal, ${ }^{21}{ }^{23} \mathrm{E}$ coli, ${ }^{45}$ and group D streptococcus endocarditis, ${ }^{23} 46$ each of which has reflected the frequency with which the infection stems from the genitourinary tract. They also indicate the now well known fact that while $S$ bovis infections respond well to penicillin those due to unspeciated enterococci and $S$ faecalis require treatment with an aminoglycoside in combination with penicillin. The nomenclature and classification of the organisms confuse the issue as does the now well known difficulty of differentiating between $S$ bovis and $S$ faecalis.?

Transient symptomless bacteraemia obviously follows alimentary and genitourinary surgical and investigative procedures not infrequently and may also occur under physiological conditions. Only an extremely small proportion of such bacteraemias progress to infective endocarditis, and among the factors influencing this development are the magnitude of the bacteraemia, the virulence of the organism, and the susceptibility of the host. The presence of a prosthetic valve, a previous episode of infective endocarditis, and a rheumatic, congenital, or degenerative cardiac abnormality play an important part, but depression of defence mechanisms by age, malignancy, alcohol dependence, diabetes, immunosuppression, hepatic or renal failure, or other serious illness are frequent contributory influences. Even so, in nearly half the 75 patients, in whom infective endocarditis was due to organisms normally resident in the gut, there was no explanation of why the bacteraemia occurred.

With regard to the prophylaxis of infective endocarditis due to bowel organisms, it is clear that such infections occur in older patients and derive 
from the genitourinary tract slightly more often than from the alimentary tract. Furthermore, only $16 \%$ of cases of infective endocarditis are due to bowel organisms, and nearly half of these are unexplained; in the face of the enormous number of operations and investigatory procedures involving the alimentary and genitourinary tracts the risk of infective endocarditis arising therefrom must be very small indeed. Nevertheless, despite the minimal danger of such procedures infective endocarditis remains an appreciable cause of morbidity and mortality, and measures for its prevention are important. Certainly, the present findings indicate that all those with known cardiac abnormalities should be given antibiotic cover for all surgical, alimentary, and genitourinary procedures and for gastroscopy. Furthermore, in view of the high proportion of older patients with normal hearts contracting infective endocarditis those in this age group should be considered for similar prophylaxis. The measures suggested by the British Society for Antimicrobial Chemotherapy ${ }^{47}$ may be appropriate, but in each patient there will be differing circumstances to be taken into account. The case for antibiotic cover is certainly as strong as that for dental procedures. In the 582 episodes of infective endocarditis, of the 329 with " $S$ viridans" infections or with negative blood cultures, only $38(12 \%)$ of those known to have a cardiac abnormality had had a dental procedure within three months of the onset of illness, and eight of the $\mathbf{3 8} \mathrm{had}$ had antibiotic prophylaxis which failed. In comparison, of the 87 patients in whom infective endocarditis was caused by bowel organisms or followed some alimentary tract investigation or operation, $15(17 \%)$ had a known cardiac abnormality, and only two received antibiotic prophylaxis at the relevant time, which failed. Nevertheless, it must be acknowledged that had prophylaxis such as we suggest been given to those of the 87 patients in whom it would have been considered appropriate at most the infective endocarditis would have been prevented in only about a fifth. Many were particularly vulnerable on account of age and other serious illness.

We thank the members of the British Cardiac Society and the fellows and members of the College who have participated in this survey.

\section{References}

1 Horder TJ. Infective endocarditis with an analysis of 150 cases and with special reference to the chronic form of the disease. $Q f$ Med 1909; 2: 289-324.

2 Cates JE, Christie RV. Subacute bacterial endocarditis: a review of 442 patients treated in 14 centres appointed by the Penicillin Trials Committee of the Medical Research Council. $Q \mathcal{F}$ Med 1951; 20: 93-130.

3 Shinebourne EA, Cripps CM, Hayward GW, Shooter
RA. Bacterial endocarditis 1956-65: analysis of clinical features and treatment in relation to prognosis and mortality. Br Heart F 1969; 31: 536-42.

4 Cherubin CE, Neu HC. Infective endocarditis at the Presbyterian Hospital in New York City from 193867. Am F Med 1971; 51: 83-96.

5 Hayward GW. Infective endocarditis: a changing disease. $\mathrm{Br}$ Med $\mathcal{F}$ 1973; ii: 706-9, 764-6.

6 Smith RH, Radford DJ, Clark RA, Julian DG. Infective endocarditis; a survey of cases in the South-East Region of Scotland, 1969-72. Thorax 1976; 31: 373-9.

7 Schnurr LP, Ball AP, Geddes AM, Gray J, McGhie D. Bacterial endocarditis in England in the 1970s: a review of 70 patients. $Q \mathcal{F}$ Med 1977; 46: 499-512.

8 Lowes JA, Hamer J, Williams G, et al. 10 years of infective endocarditis at St. Bartholomew's Hospital: analysis of clinical features and treatment in relation to prognosis and mortality. Lancet 1980; i: 133-6.

9 Moulsdale MT, Eykyn SJ, Phillips I. Infective endocarditis, 1970-79. A study of culture-positive cases in St. Thomas's Hospital. Qf Med 1980; 49: 315-28.

10 Oakley CM. Infective endocarditis. Br $\mathcal{F}$ Hosp Med 1980; 24: $232-43$.

11 Anonymous. Infective endocarditis [Leading Article]. $\mathrm{Br}$ Med F 1981; 282: 677-8.

12 Gray IR. Management of infective endocarditis. $\mathcal{f} R$ Coll Physicians Lond 1981; 15: 173-8.

13 Petersdorf RG, Goldman PL. Changes in the natural history of bacterial endocarditis. $f$ Chron Dis 1979; 32: 287-91.

14 Gray IR. The choice of antibiotic for treating infective endocarditis. $Q \mathcal{F}$ Med 1975; 44: 449-58.

15 Oram S. Clinical heart disease. 2nd ed. London: Heinemann, 1981.

16 Sipes JN, Thompson RL, Hook EW. Prophylaxis of infective endocarditis: a reevaluation. Annu Rev Med 1977; 28: 371-91.

17 Pelletier LL Jr, Petersdorf RG. Infective endocarditis: a review of 125 cases from the University of Washington Hospitals, 1963-72. Medicine (Baltimore) 1977; 56: 287313.

18 Slaughter L, Morris JE, Starr A. Prosthetic valvular endocarditis. Circulation 1973; 47: 1319-26.

19 Oakley CM. Long-term complications of valve replacement [Leading Article]. Br Med f 1982; 284: 995-7.

20 Bayliss R, Clarke C, Oakley CM, Somerville W, Whitfield AGW, Young SEJ. The microbiology and pathogenesis of infective endocarditis. Br Heart $\mathcal{F}$ 1983; 50: 513-9.

21 Mandell GL, Kaye D, Levison ME, Hook EW. Enterococcal endocarditis. Arch Intern Med 1970; 125: 258-64.

22 Bayliss R, Clarke C, Oakley C, Somerville W, Whitfield AGW. The teeth and infective endocarditis. $\mathrm{Br}$ Heart $\mathcal{f}$ 1983; 50: 506-12.

23 Ravreby WD, Bottone EJ, Keusch GT. Group D streptococcal bacteremia, with emphasis on the incidence and presentation of infections due to $S$. bovis. $N$ Engl $\mathcal{F}$ Med 1973; 289: 1400-3.

24 Crockett EJ, Batchelor TM, Corbeil J. Pregnancy and subacute bacterial endocarditis: report of a case. Obstet Gynecol 1960; 16: 93-5. 
25 Shull HJ Jr, Greene BM, Allen SD, Gunn GD, Schenker S. Bacteremia with upper gastrointestinal endoscopy. Ann Intern Med 1975; 83: 212-4.

26 Bilbao MK, Dotter CT, Lee TG, Katon RM. Complications of endoscopic retrograde cholangiopancreatography. Gastroenterology 1976; 70: 314-20.

27 Buchman E, Berglund EM. Bacteremia following sigmoidoscopy. Am Heart f 1960; 60: 863-6.

28 Le Frock JL, Ellis CA, Turchik JB, Weinstein L. Transient bacteremia associated with sigmoidoscopy. $N$ Engl F Med 1973; 289: 467-9.

29 Weinstein L. Chemoprophylaxis for bacterial endocarditis [Letter]. $N$ Engl f Med 1975; 292: 427-8.

30 Engeling ER, Eng BF, Sullivan-Sigler N, Bartlett JG, Gorbach SL. Bacteremia after sigmoidoscopy: another view [Letter]. Ann Intern Med 1976; 85: 77-8.

31 Norfleet RG, Mulholland DD, Mitchell PD, Philo J, Walters EW. Does bacteremia follow colonoscopy? Gastroenterology 1976; 70: 20-1.

32 Dickman MD, Farrell R, Higgs RH, et al. Colonoscopy associated bacteremia. Surg Gynecol Obstet 1976; 142: 173-6.

33 Roses DF, Richman H, Localio SA. Bacterial endocarditis associated with colorectal carcinoma. Ann Surg 1974; 179: 190-1.

34 Le Frock JL, Ellis CA, Klainer AS, Weinstein L. Transient bacteremia associated with barium enema. Arch Intern Med 1975; 135: 835-7.

35 McCloskey RV, Gold M, Weser E. Bacteremia after liver biopsy. Arch Intern Med 1973; 132: 213-5.

36 Le Frock JL, Ellis CA, Turchik JB, Zawacki JK, Weinstein L. Transient bacteremia associated with percutane- ous liver biopsy. $\mathcal{F}$ Infect Dis 1975; 131 (suppl): S104-7.

37 Ashby EC, Rees M, Dowding $\mathrm{CH}$. Prophylaxis against systemic infection after transrectal biopsy for suspected prostatic carcinoma. Br Med F 1978; ii: 1263-4.

38 Klein RS, Recco RA, Catalano MT, Edberg SC, Casey JI, Steigbigel NH. Association of Streptococcus bovis with carcinoma of the colon. N Engl F Med 1977; 297: 800-2.

39 Noble CJ, Uttley AHC, Falk RH, Richardson PJ. Streptococcus bovis endocarditis and colonic cancer [Letter]. Lancet 1978; i: 766.

40 Hossenbux K, Dale BAS, Walls ADF, Lawrence JR. Streptococcus bovis endocarditis and colonic carcinoma: a neglected association. Br Med f 1983; 287: 21.

41 Murray HW, Roberts RB. Streptococcus bovis bacteremia and underlying gastrointestinal disease. Arch Intern Med 1978; 138: 1097-9.

42 Scully RE, Mark EJ, McNeely BU, eds. Case records of the Massachusetts General Hospital. Case 34-1982. N Engl f Med 1982; 307: 543-9.

43 Dunham WR, Simpson JH, Feest TG, Cruickshank JG. Streptococcus bovis endocarditis and colorectal disease [Letter]. Lancet 1980; i: 421-2.

44 Lerner PI, Weinstein L. Infective endocarditis in the antibiotic era. $N$ Engl $\mathcal{Y}$ Med 1966; 274: 199-206.

45 Hansing CE, Allen VD, Cherry JD. Escherichia coli endocarditis. Arch Intern Med 1967; 120: 472-7.

46 Moellering RC Jr, Watson BK, Kunz LJ. Endocarditis due to group D streptococci. Am 7 Med 1974; 57: 239 50.

47 British Society for Antimicrobial Chemotherapy. The antibiotic prophylaxis of infective endocarditis. Report of a Working Party. Lancet 1982; ii: 1323-6. 